\title{
Low Energy Scanning Transmission Electron Microscope
}

\author{
Ilona Müllerová, Eliška Mikmeková, Ivo Konvalina and Luděk Frank
}

Institute of Scientific Instruments, AS CR, Brno, Czech Republic.

This paper describes how to obtain high resolution in a Low Energy Scanning Transmission Electron Microscope (LESTEM) down to units of $\mathrm{eV}$ with a spot size of units of $\mathrm{nm}$. The imaging of graphene in the transmitted signal is shown as one application example.

The transmission electron microscope and its scanning version (STEM) operating in the energy range of hundreds of $\mathrm{keV}$ are widely used instruments for the imaging of thin specimens at the atomic level. Recently, STEM attachments to a standard scanning electron microscope (SEM) have become available [1], and at present the majority of modern SEM devices have the possibility of collecting transmitted signals, both in bright and dark fields. These assemblies are usually operated at tens or even units of $\mathrm{keV}$. Resolution at the atomic level is not yet available, but a spot size below $1 \mathrm{~nm}$ has been demonstrated. The increase in contrast, especially for light materials, polymers or organic samples, is an important advantage, along with the possible combination of multiple image signals [2].

To operate down to units of eV with a small primary spot size, a cathode lens with a biased specimen was introduced into the SEM [3] (Fig. 1). The reflected signal, secondary and backscattered (BSE) electrons, is usually collected by detectors situated above the specimen. When inserting a detector below the specimen, the transmitted electron (TE) signal can be used for imaging down to zero energy (Fig. 2). The range of units of $\mathrm{eV}$ is of interest because the inelastic mean free path, primarily responsible for the electron penetration, begins to grow below approximately $50 \mathrm{eV}$, see for example [5]. The transmitted signal is collimated to the optical axis and accelerated to the detector similarly to how it works with the reflected signal [4]. Several detection systems have been proven.

Various graphene flakes have been tested and maximum transmittance was measured at $5 \mathrm{eV}$ for single layer graphene (1-LG) [4]. One recognized method for the diagnostics of graphene is Raman spectroscopy [6]. However, we have found flakes exhibiting a Raman spectrum corresponding to 1-LG ( $G$ ' peak near to $2,700 \mathrm{~cm}^{-1}$ and well fitted with a single Lorentzian, and a ratio of $\mathrm{G}$ to $\mathrm{G}$ ' peaks near 0.5 ) that can be identified with the LESTEM as an aggregate of smaller flakes of various thicknesses [7] (Fig. 3). This is due to the higher contrast of the LESTEM method and its better lateral resolution [8].

\section{References:}

[1] V Morandi and PG Merli, Journal of Applied Physics 101 (2007), p.1.

[2] LF Drummy et al, Ultramicroscopy 99 (2004), p. 247.

[3] I Müllerová and L. Frank, Adv. Imaging \& Electron Phys. 128 (2003), p.309.

[4] I Müllerová et al, Ultramicroscopy 119 (2012), p. 78.

[5] HJ Fitting et al, Journal of Electron Spectroscopy and Related Phenomena 119 (2001), p. 35.

[6] LM Malard et al, Physics Reports 473 (2009), p. 51.

[7] E Mikmeková et al, Journal of Microscopy, submitted.

[8] The authors acknowledge funding from the Technology Agency of the Czech Republic (project no. TE01020118). 

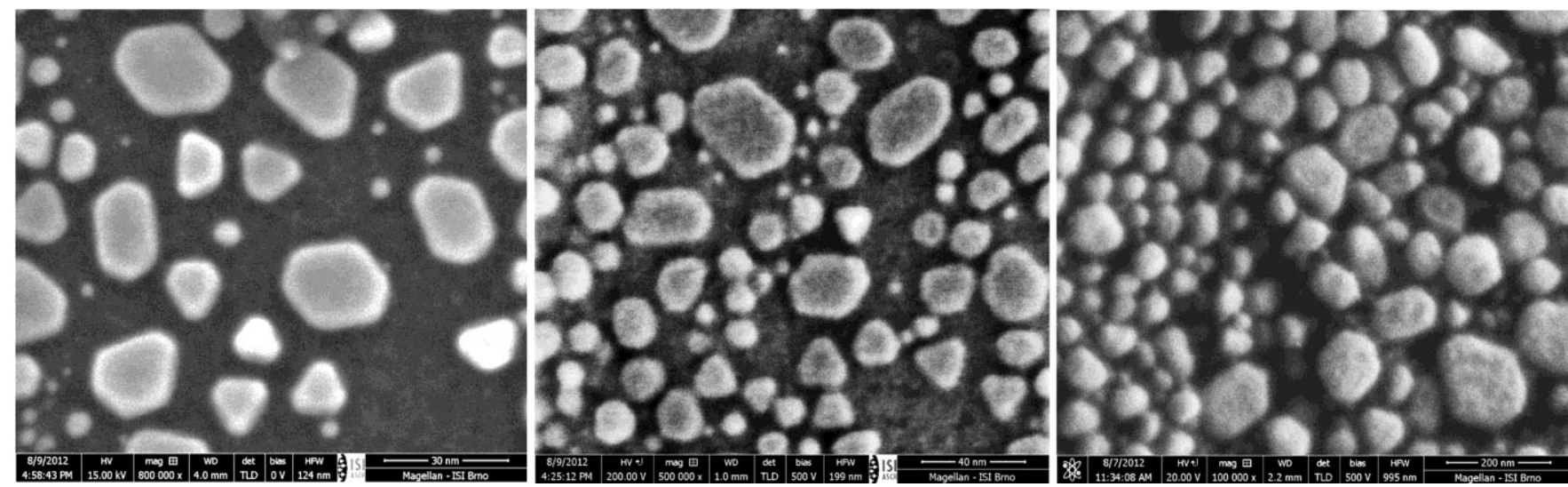

Figure 1. The gold on carbon particles imaged in a SEM fitted with a cathode lens. The calculated resolution is $0.5 \mathrm{~nm}$ at $15 \mathrm{keV}$ (left), $0.8 \mathrm{~nm}$ at $200 \mathrm{eV}$ (middle) and $4.5 \mathrm{~nm}$ at $20 \mathrm{eV}$ (right).
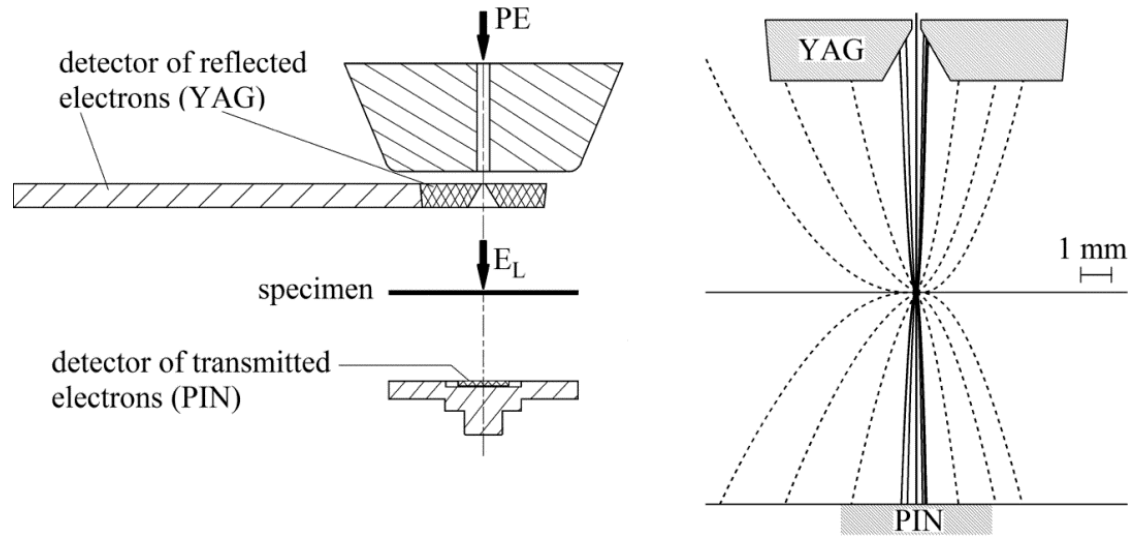

Figure 2. Left: the cathode lens assembly. Right: an example of the trajectories of the reflected and transmitted signal electrons. The initial energies of electrons are $5 \mathrm{eV}$ (full lines) and $300 \mathrm{eV}$ (dashed lines) in the right half-space, and $10 \mathrm{eV}$ (full lines) and 1,000 eV (dashed lines) in the left half-space. The initial polar angles are $0^{\circ}, 30^{\circ}, 60^{\circ}$, and $90^{\circ}$ with respect to the optical axis. The electrostatic field strength between the detectors and the specimen is $5 \mathrm{keV} / 7 \mathrm{~mm}$.

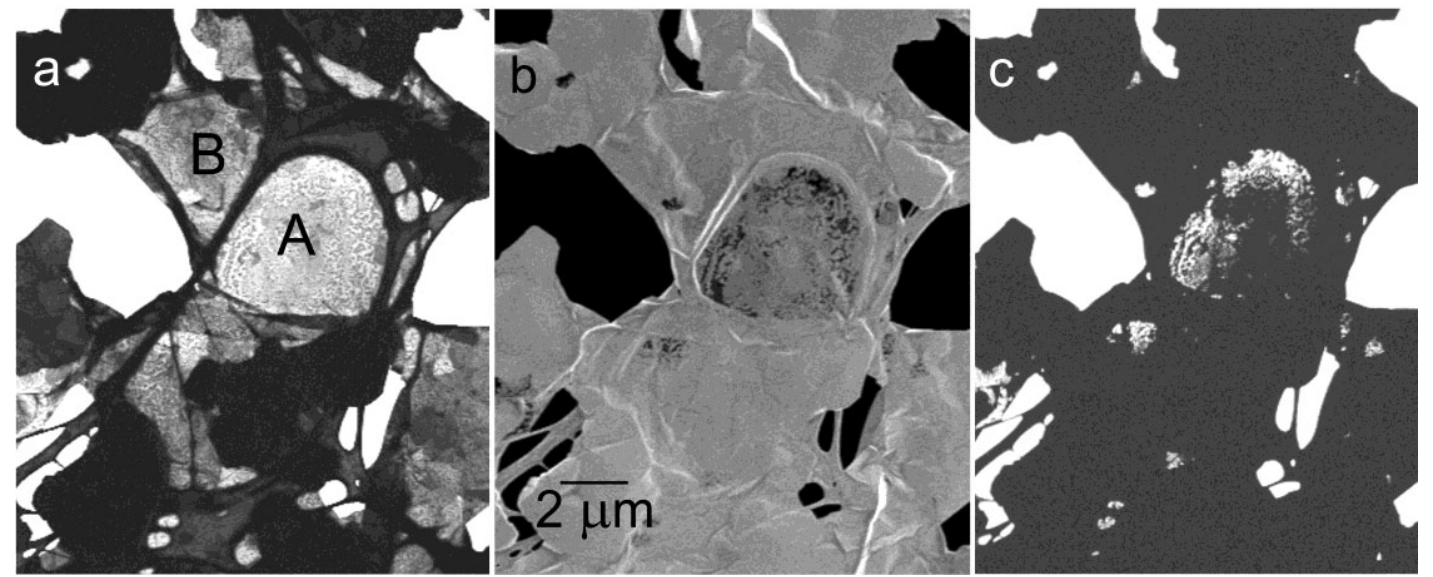

Figure 3. Low energy electron micrographs of graphene flakes; Raman spectroscopy identifies site A as 1-LG. TE image at 1,000 eV (a), BSE image at 1,000 eV (b), TE image at $5 \mathrm{eV}$ (c). 